\title{
Avaliação psicológica online: considerações a partir da pandemia do novo coronavírus (COVID-19) para a prática e o ensino no contexto a distância
}

\author{
Psychological assessment online: repercussions of the new \\ coronavirus (COVID-19) pandemic on remote practice and \\ distance teaching
}

Aline Riboli MARASCA ${ }^{1}$ (iD) 0000-0002-9928-3148

Denise Balem YATES ${ }^{2}$ (iD) 0000-0002-0879-9270

Andreia Mello de Almeida SCHNEIDER' ${ }^{1}$ (iD) 0000-0003-0767-2005

Luan Paris FEIJÓ3 ${ }^{\text {ID }}$ 0000-0002-7587-3987

Denise Ruschel BANDEIRA' 1 (iD) 0000-0001-9867-2718

\section{Resumo}

As restrições impostas pelo distanciamento social decorrente da pandemia do novo coronavírus exigiram adaptações dos psicólogos a uma nova realidade de trabalho que privilegia atividades remotas. O ensino e a prática em Avaliação Psicológica foram algumas das áreas afetadas, demandando que psicólogos, conselho profissional e sociedades científicas discutam diretrizes para o contexto da pandemia. Também, ainda que exista um aumento de cursos à distância, são apontadas restrições para o ensino de técnicas psicológicas em ambiente online. Visto as mudanças no cenário de

$\cos$

${ }^{1}$ Universidade Federal do Rio Grande do Sul, Instituto de Psicologia, Programa de Pós-Graduação em Psicologia. Porto Alegre, RS, Brasil. R. Ramiro Barcelos, 2600, Santa Cecília, 90035-003, Porto Alegre, RS, Brasil. Correspondência para/Correspondence to: D.R. BANDEIRA. E-mail: <deniserbandeira@gmail.com>.

${ }^{2}$ Universidade Federal do Rio Grande do Sul, Instituto de Psicologia, Centro Interdisciplinar de Pesquisa e Atenção à Saúde. Porto Alegre, RS, Brasil.

${ }^{3}$ Universidade do Vale do Rio dos Sinos, Escola de Saúde, Programa de Pós-Graduação em Psicologia. São Leopoldo, RS, Brasil. Suporte: Conselho Nacional de Desenvolvimento Científico e Tecnológico e Coordenação de Aperfeiçoamento de Pessoal de Nível Superior (Código de Financiamento 001).

$\boldsymbol{\nabla} \mathbf{v} \boldsymbol{V}$

Como citar este artigo/How to cite this article

Marasca, A. R., Yates, D. B., Schneider, A. M. A., Feijó, L. P., \& Bandeira, D. R. (2020). Avaliação psicológica online: considerações a partir da pandemia do novo coronavírus (COVID-19) para a prática e o ensino no contexto a distância. Estudos de Psicologia (Campinas), 37, e200085. https://doi.org/10.1590/1982-0275202037e200085 
trabalho e a necessidade de adaptação à situação atual, este estudo busca discutir a viabilidade de processos de Avaliação Psicológica online e apontar direções para seu aperfeiçoamento. Procura-se também apresentar possibilidades para ensino e supervisão a distância. Discutem-se evidências científicas e regulamentações nacionais e internacionais que embasam essas práticas. Por fim, reforça-se a necessidade do desenvolvimento de tecnologias que permitam conduzir o processo de maneira ética e segura.

Palavras-chave: Educação a distância; Epidemias; Prática psicológica; Tecnologia da Informação.

\begin{abstract}
Given the restrictions imposed by social distancing as a result of the new coronavirus pandemic, psychologists are dealing with a new working reality that is shifting the focus towards remote activities. Teaching and practice in psychological assessment are just two of the areas that have been affected, requiring psychologists, professional bodies and scientific societies to discuss guidelines relating to pandemic scenarios. Although there has been an increase in distance learning courses, restrictions have been recommended for the teaching of psychological assessment techniques in the online environment. Given the changes in the work scenario and the need to adapt to the current situation, the aim of this study is to discuss the viability of online psychological assessment processes and to devise guidelines for their improvement. It also seeks to propose options for distance education and supervision. Scientific tests and national and international regulations that support these practices are discussed. Lastly, the need to develop technologies that will allow the process to be ethically and safely conducted is emphasized.
\end{abstract}

Keywords: Education distance; Epidemics; Psychological practice; Information technology.

A Avaliação Psicológica (AP) realizada por meios de Tecnologias da Informação e da Comunicação (TIC) foi uma das atividades regulamentadas como possibilidade de atuação do psicólogo a partir da Resolução $n^{\circ}$ 11/2018 (Conselho Federal de Psicologia [CFP], 2018a). Nela está prevista a AP, considerando o uso de testes psicológicos com padronização e normatização específicas para a modalidade online, e a supervisão entre profissionais, desde que para ambos os serviços os psicólogos tenham registro ativo no respectivo Conselho Regional. A partir do que é descrito no documento, entende-se que a AP online pode ser conduzida em diferentes contextos, desde que atenda às premissas e restrições regulamentadas pelas resoluções vigentes. Entre as restrições, estão o atendimento de pessoas ou grupos em situação de urgência e emergência e de casos de violação de direitos ou de violência, o que foi modificado temporariamente pela Resolução nº 04/2020 (CFP, 2020a). O atendimento de crianças e adolescentes pode ocorrer mediante o consentimento de um dos responsáveis legais, desde que ponderada sua viabilidade técnica (CFP, 2018a). Em decorrência das medidas de distanciamento social impostas pela Coronavirus Disease 2019 (COVID-19), a Resolução CFP n 04/2020 também buscou facilitar o cadastramento de psicólogos para a prestação de serviços online (CFP, 2020a).

Internacionalmente, desde o final da década de 1990, são utilizadas as TIC para a avaliação das funções cognitivas, na época, realizada por telefone (Desmond, Tatemichi, \& Hanzawa, 1994). Nos Estados Unidos, diretrizes que incluem AP e testagem estão vigentes desde 2013 (American Psychological Association [APA], 2013) e, em decorrência da COVID-19, novas orientações que abrangem a segurança dos instrumentos, a qualidade dos dados obtidos, os intervalos de confiança a serem utilizados e os padrões éticos e técnicos foram divulgadas (APA, 2020). Nesse contexto, diversos estudos elencaram as vantagens do atendimento psicológico remoto, entre eles o acesso a serviços que poderiam não estar disponíveis em determinadas regiões e a redução de custos, como de deslocamento e de espaço físico (Martin, Millan, \& Campbell, 2020; McCord, Bernhard, Walsh, Rosner, \& Console, 2020). Além disso, pode ser uma solução quando o atendimento presencial não é viável, como, por exemplo, a vigência da determinação de distanciamento social em virtude da pandemia. 
No entanto, no Brasil, a utilização das TIC nas distintas áreas da psicologia é incipiente quando comparada ao contexto internacional. Verifica-se uma carência na experiência e formação dos profissionais brasileiros para utilização das TIC na prática clínica (Feijó, Silva, \& Benetti, 2018; Hallberg \& Lisboa, 2016). Considera-se que, na área de AP, devido à recência da Resolução, essa realidade não seja diferente.

Para se ter uma ideia da carência, são apresentadas duas histórias reais relatadas aos autores. A primeira é de um psicólogo clínico, que realiza psicodiagnóstico. Em uma entrevista para uma atividade acadêmica, ele relatou que, em razão da possibilidade de AP online, adaptou um instrumento de avaliação de sintomas depressivos, restrito a psicólogos e com parecer favorável no Sistema de Avaliação de Testes Psicológicos (SATEPSI) somente para aplicação presencial, para um formulário online e gratuito, a fim de utilizá-lo com seus pacientes. A segunda história é de uma psicóloga, que atua em vara de família em uma cidade do interior do Mato Grosso, a 750 km de distância de Cuiabá, sendo esta a cidade de referência para que à profissional pudesse adquirir testes psicológicos, realizar cursos e supervisão. Durante um congresso em Porto Alegre, afirmou que, assim como ela, suas colegas se sentem desamparadas nas avaliações relacionadas a disputa de guarda, sem material adequado, sem orientação e supervisão na cidade onde residem e atuam como psicólogas.

Essas duas histórias são recortes do que é perceptível na prática profissional: a falta de treinamento adequado e de instrumentalização profissional para atuação nesse contexto. No primeiro caso, vemos o psicólogo interferir na validade e fidedignidade do instrumento, à medida que utiliza um teste psicológico de aplicação presencial de maneira online, o que pode comprometer os resultados, ferindo o previsto no Código de Ética Profissional do Psicólogo, na Resolução do CFP n 11/2018 e os direitos autorais da publicação. No segundo caso, é possível notar que a produção do conhecimento científico, muitas vezes vinculada aos grandes centros urbanos, poderia ser disseminada em cidades do interior por meio de atividades remotas. Além disso, a construção de plataformas para cursos, material de apoio e supervisão a distância conseguiriam alcançar esses profissionais, permitindo que realizassem um trabalho de melhor qualidade.

É reconhecido que o uso de TIC amplia as possibilidades de atuação profissional e auxilia no desenvolvimento de estratégias para enfrentar os possíveis obstáculos da prática (Feijó et al., 2018). No entanto, diferentemente do volume de estudos sobre processos psicoterápicos realizados em ambiente virtual, a literatura ainda é escassa ao descrever a prática da AP online. Por consequência, diante das abruptas medidas de distanciamento social impostas pela COVID-19, os profissionais que atuam com AP se viram com processos presenciais interrompidos e pouca qualificação para atender a essas e a novas demandas de maneira remota. Com o intuito de contribuir para boas práticas na área, o presente estudo pretende discutir a viabilidade de processos de AP online e apontar direções para seu aperfeiçoamento, tomando como base a literatura científica e diretrizes nacionais e internacionais. Além disso, busca-se apresentar possibilidades para o ensino e a supervisão em AP na modalidade a distância.

\section{A prática da Avaliação Psicológica online}

Assim como os processos de AP realizados presencialmente, a prática por meio das TICs deve seguir as especificações técnicas e éticas próprias da AP (CFP, 2018b). Contudo, sua viabilização requer a adição de cuidados específicos para que ocorra de maneira segura, eficaz e ética no ambiente virtual (McCord et al., 2020). Com base nas diretrizes do CFP, orientações de órgãos internacionais, relatos de pesquisa e observações sobre como é a AP atualmente no Brasil, alguns apontamentos e reflexões são necessários a fim de criar possibilidades para sua efetivação no território nacional. 
Antes de iniciar a avaliação online, é fundamental conhecer a demanda e a pertinência para um processo em modalidade remota. A Resolução no 11/2018 (CFP, 2018a) indica as premissas e restrições para que ela ocorra. Porém, além do exposto pela Resolução, outras situações são passíveis de reflexão por parte do profissional. Em especial, devem ser consideradas as características da pessoa a ser atendida, como idade, condições físicas e cognitivas, fatores culturais e outros dados como familiaridade com o uso de tecnologia, disponibilidade de acesso a aparelhos eletrônicos e qualidade da conexão à internet (Luxton, Pruitt, \& Osenbach, 2014).

Por exemplo, uma pessoa poderia apresentar algumas limitações para o processo avaliativo online, como ter dificuldade em manejar as TIC, apresentar fadiga ou desconforto físico causado pelo uso da tecnologia, evidenciar alguma restrição que a impossibilite de uma total compreensão auditiva, visual ou de agilidade motora. Ainda, a ansiedade para lidar com computadores e outros recursos pode afetar as respostas emitidas em um teste psicológico nesse ambiente (Eckford \& Barnett, 2016). Pondera-se que algumas restrições físicas e cognitivas não necessariamente irão impossibilitar a realização da avaliação. Contudo, essas devem ser conhecidas a fim de que sejam empregadas técnicas mais adequadas ou que contem com o suporte eventual de uma terceira pessoa no processo (Luxton et al., 2014), o que no Brasil ainda carece de pesquisas.

Outras características do caso poderão ser determinantes para não adotar a opção online. Na área clínica, a presença de certos diagnósticos de transtornos mentais, o tipo de sintoma apresentado pelo paciente (como sintomas psicóticos) e o uso de substâncias, podem ser indicadores de risco para processos conduzidos nessa modalidade (APA, 2013). No contexto judicial ou em outros de avaliação compulsória, o aspecto de não voluntariedade do avaliando para a realização do procedimento é uma condição que pode apresentar implicações para o resultado pretendido (CFP, 2020b). Nesse sentido, antes de iniciar a avaliação, o psicólogo deve ponderar os benefícios e possíveis prejuízos da prática.

A análise de benefícios e riscos é um dos aspectos abordados no contrato a ser estabelecido com o avaliando. De modo geral, em um processo de AP, é desejável que seja esclarecido o que é AP, quais os benefícios para o avaliado, como ela será conduzida (em relação à duração, ao contato com informantes e aos documentos derivados do processo), além de combinações acerca de horários e honorários, se aplicável. No contexto virtual, deve ser reforçada a ênfase no sigilo, alertando para a não autorização de gravações de áudio ou vídeo. Além de inteirar quais informações serão repassadas e quem terá acesso a elas, o psicólogo deverá ter clareza sobre a segurança do ambiente virtual que utiliza como mediador do processo de avaliação (CFP, 2018a).

O psicólogo também necessita atentar para outros aspectos do setting que tendem a ser mais controlados quando a avaliação ocorre presencialmente. É importante orientar que o avaliando esteja em um ambiente confortável, com privacidade, sem distrações e seguro de que não será interrompido (APA, 2013). Esse aspecto é relevante não só para maior garantia do sigilo das informações, mas também para assegurar maior precisão na condução dos testes psicológicos. Para tanto, sugere-se o uso de um contrato por escrito, em que essas orientações, além de explicadas, sejam formalmente aceitas pelo avaliando, a fim de resguardar o psicólogo que conduz o processo e a validade das informações resultantes da avaliação (Lustgarten \& Elhai, 2018)

Apesar de termos poucos dados de pesquisa acerca do processo de AP online, o uso de testes psicológicos em ambiente virtual e suas diferenças em relação à versão de lápis e papel é expresso na literatura (APA, 2020). A construção ou adaptação de um instrumento psicológico envolve um rigoroso trabalho de pesquisa e reunião de evidências que embasam a utilidade de seus resultados e interpretações para serem aplicados em determinados contextos (Andrade \& Valentini, 2018). Dessa forma, apenas exportar os estímulos de um instrumento para ferramentas online não garante que eles sejam visualizados pelo avaliado da mesma forma como seriam em um atendimento presencial, por exemplo, em relação às suas cores, ao tamanho ou a outras 
características que o definem. Sendo assim, como estabelecer se as respostas do paciente são compatíveis à interpretação preconizada no manual se há uma variação nos estímulos empregados?

Por sua vez, escalas e inventários administrados pela internet tendem a ter alta correlação com suas versões em lápis e papel, mas não é possível assumir em todas as situações que suas propriedades psicométricas serão idênticas (Pritchard, Stephan, Zabel, \& Jacobson, 2017). Alguns argumentos para isso são possíveis efeitos de desinibição e mudanças na motivação dos respondentes em ambiente online, diferentemente da amostra comparativa que esteve em aplicação presencial (Reips, Buchanan, Krantz, \& McGraw, 2016). Também é importante reiterar que é vedado ao psicólogo interferir na validade e fidedignidade de instrumentos e técnicas psicológicas (CFP, 2005), o que seria o caso de usar em modo online um teste que não possui evidências de validade para tal método de aplicação.

Diretrizes estabelecidas pela International Test Comission (ITC, 2005; 2017) e nas Standards for Educational and Psychological Testing (American Educational Research Association [AERA], American Psychological Association [APA], \& National Council on Measurement in Education [NCME], 2014) apontam especificidades para o desenvolvimento ou adaptação de instrumentos mediados por TIC no que diz respeito à padronização, à normatização e às evidências de validade dos resultados extraídos neste contexto. Esses cuidados também são traduzidos na Resolução n 11/2018 (CFP, 2018a), que enfatiza a necessidade de estudos de padronização e normatização específicos para a modalidade remota.

Até maio de 2020, a lista de testes favoráveis do SATEPSI para aplicação online/remota contava com quatro instrumentos, sendo que esses avaliam atenção, personalidade e interesses pessoais. Além desses, outros 20 atualmente são listados no SATEPSI como instrumentos que permitem a aplicação informatizada, ou seja, por intermédio do computador, mas presencialmente com o avaliando. A Nota Técnica n 07/2019 (CFP, 2019a) pontua que o formato de aplicação informatizada não equivale à aplicação online/remota, que se refere a aplicação a distância do teste. Desse modo, entende-se que cabe ao psicólogo a análise e o estudo do manual do teste psicológico para identificar se a padronização permite o uso online/remoto, ou se permite a aplicação informatizada, mas presencial.

Outros aspectos são pontuados como limitações em relação à utilização dos testes psicológicos na modalidade online. Entre eles, o baixo controle do psicólogo acerca do ambiente de testagem e as possíveis interrupções devido à qualidade da conexão à internet (APA, AERA, \& NCME, 2014). Em tarefas dependentes de tempo, por exemplo, uma falha na rede de internet pode comprometer a utilização do resultado. Apesar de orientações para o avaliando sobre tais questões, estas ainda podem ser inevitáveis em certas ocasiões.

Em outra direção, são reforçados os pontos positivos da aplicação de testes psicológicos mediados por TIC. A tecnologia pode auxiliar na precisão de algumas medidas, como o tempo de reação ou o rastreio do movimento ocular (Poletti et al., 2017; Schmand, 2019). Em determinados contextos, o uso de testes em versão informatizada pode aumentar a interação com o examinando, apresentar maior riqueza dos estímulos, fornecer maior segurança e rapidez para o armazenamento dos protocolos respondidos, assim como facilitar a pontuação e interpretação dos resultados (Oliveira, Bandeira, \& Giacomoni, 2019). Assim, é reforçada a importância da pesquisa para o desenvolvimento a adaptação de tais instrumentos, a fim de contribuir com o trabalho do psicólogo e ampliar suas possibilidades de atuação. No contexto nacional, algumas iniciativas nesse sentido estão sendo delineadas por editoras de testes psicológicos e outras empresas já oferecem plataformas para facilitar a integração dos dados dos instrumentos aplicados e a elaboração de documentos decorrentes da AP.

Um dos principais produtos da AP é a emissão de documentos psicológicos. No entanto, até o momento, não são fornecidas diretrizes particulares para a produção de documentos derivados da AP online. No contexto de distanciamento social decorrente da COVID-19, o CFP emitiu recomendações sobre a elaboração de documentos psicológicos para o Poder Judiciário, indicando que estes sejam datados e 
devam situar que foram produzidos no período de pandemia (CFP, 2020b). Também pode ser pertinente informar que a avaliação foi realizada em formato online e em consonância com as resoluções específicas do CFP para a prática.

De forma geral, a Resolução n 06/2019 do Conselho Federal de Psicologia para elaboração de documentos (CFP, 2019b), bem como o Código de Ética Profissional do Psicólogo (CFP, 2005), enfatizam o cuidado acerca do conteúdo confidencial do material. Assim, é importante que o psicólogo possa se cercar de procedimentos que aumentem a garantia da segurança das informações compartilhadas no documento. Entre as considerações possíveis para a prática, estão o uso de arquivos em formato protegido e a utilização de certificação digital de assinaturas. Com a popularização da atividade, é provável o desenvolvimento de plataformas digitais mais especializadas para compartilhamento desses documentos a partir de usuário registrado e senha fornecidos pelo profissional para o solicitante. Assim como as plataformas para videoconferência, recomenda-se que esses aplicativos utilizem criptografia de ponta a ponta ou sejam certificadas pela Health Insurance Portability and Accountability Act (HIPAA), que propõe padrões mínimos de proteção de confidencialidade de informações de saúde, ou pela Health Information Technology for Economic and Clinical Health Act, a qual expandiu o escopo da HIPAA (Twist, Hertlein, \& Haider, 2016).

Por fim, torna-se importante realizar algumas ponderações acerca do estabelecimento dos honorários. Um argumento frequentemente lançado como vantagem dos atendimentos psicológicos online é a redução dos custos para sua viabilização. Por um lado, para o psicólogo, é abonada a onerosa manutenção de uma sala de consultório, seja de um imóvel próprio ou locado, assim como os gastos com transporte, estacionamento privado, entre outros. Por outro lado, é possível notar que outros custos acabam se somando para dar condições ao trabalho remoto. Entre eles, podemos citar a mensalidade para o uso de plataformas que garantam a segurança das informações, possíveis taxas transacionais que incidem sobre o valor de cada consulta, uma boa rede de internet e um computador com qualidade de áudio e vídeo. Da mesma forma, para assegurar a validade dos documentos emitidos em formato online, pode-se somar o custo de confecção e atualização da assinatura digital certificada. Além disso, são mantidos os custos da aquisição de manuais de testes psicológicos e seus protocolos online. Assim, tais valores devem ser examinados no momento da fixação dos honorários e talvez sugiram que é ilusória a expectativa de que o trabalho remoto gere uma redução de custos para o avaliador.

\section{O ensino da Avaliação Psicológica em contexto de educação a distância}

Há alguns anos se discute a formação e o ensino da AP no Brasil. A carência de uma formação sólida se percebe de modo concreto quando mais de $60 \%$ dos processos éticos conduzidos no CFP se referem a infrações relativas à AP e aos documentos (Zaia, Oliveira, \& Nakano, 2018). Segundo as Diretrizes Curriculares Nacionais para os cursos de graduação em Psicologia, o curso deve seguir sendo generalista (Ministério da Educação, 2019). Isso reforça a necessidade de que aqueles que desejam atuar na área de AP devem buscar formação complementar e supervisão antes de aceitar conduzir um trabalho, pois é uma especialidade do psicólogo que requer ampla fundamentação em teorias psicológicas, domínio no uso de testes psicológicos, instrumentos e técnicas, cuidados éticos e habilidade para contextualização dos resultados e integração dos dados coletados durante o processo (Bueno \& Peixoto, 2018; Gouveia, 2018; Muniz, 2018; Schneider, Marasca, Dobrovolski, Muller, \& Bandeira, 2020).

Em meio a uma formação carente, surge a possibilidade do ensino a distância. Cursos de extensão e aulas em cursos de graduação e pós-graduação vêm mudando com o uso das TIC. Já se previa que a tecnologia seria um componente integrante da formação em psicologia (Deane, Gonsalvez, Blackman, Saffioti, 
\& Andresen, 2015), mas, com o surgimento da necessidade do distanciamento social imposta pela COVID-19, o ensino online da AP é uma prática que vem se consolidando e deve permanecer mesmo após a pandemia.

O Código de Ética Profissional do Psicólogo determina, no Art.18, que: "o psicólogo não divulgará, ensinará, cederá, emprestará ou venderá a leigos instrumentos e técnicas psicológicas que permitam ou facilitem o exercício ilegal da profissão" (CFP, 2005). O psicólogo, professor de psicologia que ministrará as aulas relacionadas à AP, não deve, assim, disponibilizar na internet materiais que facilitem a disseminação de conteúdos relacionados a testes psicológicos.

Isso ratifica, mesmo no ensino presencial, a não disponibilização de apresentações em PowerPoint ou Portable Document Format (PDF), por exemplo, em ambientes de ensino como o Modular Object-Oriented Dynamic Learning Environment (Moodle) ou e-mail, com conteúdos restritos a psicólogos e estudantes de psicologia, que permitam que o aluno baixe a informação e a compartilhe com quem achar conveniente. Em tempos de distanciamento social, em que os professores precisaram adaptar suas aulas para um formato totalmente online, aulas, mesmo que transmitidas ao vivo, podem ser gravadas para revisão do conteúdo, fazendo com que o professor não tenha controle dos rumos que essa gravação pode tomar, o que acaba por ferir o Art. 18 do Código de Ética Profissional do Psicólogo mencionado. Embora essas situações possam acontecer também no contexto presencial, quando apresentados na prática remota, a disseminação deste conteúdo pode ser ainda maior, tendo em vista a facilidade de já estar disponível de modo digital.

Tais riscos de divulgação de conteúdo exclusivo ocorrem também em modalidades de ensino presencial, pois os alunos podem gravar em áudio ou vídeo as aulas, ou ainda fotografar as projeções sem que o professor perceba. Dessa forma, sugerem-se algumas estratégias de cuidado específicas para essas questões. Inicialmente, antes de ministrar aulas com conteúdos exclusivos (presencial ou remotamente), é importante discutir com os alunos os danos que podem advir da não observância do Art. 18 do Código de Ética Profissional do Psicólogo (CFP, 2005). Depois, solicitar a assinatura (ou concordância por alguma forma digital legalmente válida) de um Termo de Compromisso estabelecendo que o aluno se comprometa a não divulgar imagens, áudios ou vídeos das aulas sob quaisquer formas. Outra estratégia simples indicada aos professores de AP é inserir nos slides e materiais impressos uma marca d'água indicando que o material foi elaborado somente para fins didáticos e não pode ser distribuído. Essas sugestões, individualmente, podem não garantir efetivamente que os alunos não compartilhem as informações de trato exclusivo dos psicólogos. Contudo, se aplicadas simultaneamente, podem aumentar a consciência de uma postura ética, um aspecto fundamental de ser apresentado na formação do profissional.

Para os cursos da área de AP, idealmente, poderiam ser utilizadas plataformas criptografadas, além de senha com validade por um período restrito (e.g., 60 dias). Tal limitação reduz a chance de compartilhamento de dados, bem como garante a atualidade das informações. Cursos com acesso "vitalício" correm o risco de se tornarem obsoletos em uma área em que frequentemente ocorrem atualizações de padronização, normas, estudos de precisão e evidências de validade, além de revisões nas Resoluções do Conselho Federal de Psicologia, gerando o risco de o aluno acessar materiais obsoletos.

\section{A prática de supervisão em ambiente virtual}

A supervisão pode ser definida como uma formação intensiva ou treinamento, focada em casos clínicos representativos da realidade profissional e que provê apoio, dirige e orienta o trabalho de colegas por um profissional da área da saúde qualificado ou com experiência comprovada (Wright, Brabender, \& Pade, 2019). No caso da supervisão em AP, esta deve ser administrada por psicólogo com experiência na área (Wright et al., 2019), podendo ser complementada por discussões e treinamento com profissionais de outras áreas da saúde. 
As competências clínicas da AP, similares à psicoterapia, envolvem técnicas de entrevista, conhecimento de princípios e padrões éticos, competências multiculturais, habilidades diagnósticas e habilidades interpessoais. Há ainda competências específicas à área da AP, tais como conhecimentos psicométricos, seleção de instrumentos, administração e levantamento de testes, interpretação e integração de achados, desenvolvimento de indicações terapêuticas, escrita de documentos e habilidades de devolução (Vannucci, Whiteside, Saigal, Nichols, \& Hileman, 2017).

Algumas das técnicas de supervisão utilizadas em AP são a supervisão individual e coletiva, a discussão de casos, a checagem da aplicação e levantamento de testes, a leitura dos relatos de atendimento, a supervisão da escrita dos documentos, o acompanhamento da avaliação em sala de espelhos, gravação em áudio e/ou vídeo das avaliações (Yates, 2016). As formas de supervisão online em psicologia, por sua vez, permitem o uso de recursos como videoconferência (individual ou em grupo), compartilhamento de dados na nuvem (textos, áudios e vídeos) e o uso de softwares de seguimento dos resultados clínicos (Hagstrom \& Maranzan, 2019). Tais recursos sugerem a possibilidade de transposição da supervisão presencial em AP para a supervisão online sem maiores dificuldades. Contudo, alguns cuidados devem ser tomados para a garantia do sigilo e segurança dos dados compartilhados pela internet, bem como para a manutenção de um processo de supervisão ético e com qualidade.

Situações como a pandemia da COVID-19 demonstram como a supervisão a distância pode vir a ser útil para os profissionais da área da AP. Além de períodos de distanciamento social, a supervisão online permite aproximar profissionais e supervisores de regiões distantes, bem como garantir a manutenção da supervisão em momentos em que o contato presencial não possa ocorrer. Pode-se discutir se haveria um custo menor para a oferta de supervisão online, em virtude deste poder ser oferecida a partir da residência do supervisor, sem exigir um consultório ou outro local de trabalho. Entretanto, assim como destacado na seção da prática da AP deste artigo, outros custos podem se somar quando se ministra a supervisão remota como, por exemplo, a adoção de plataformas criptografadas para evitar que as informações discutidas entre os profissionais sejam hackeadas por terceiros.

É importante considerar que a supervisão a distância também gera alguns desafios para o supervisor, como a aparente redução do controle do processo, ao não poder manusear os protocolos aplicados pelo supervisionando (que geralmente coloca uma "pilha" de materiais sobre a mesa, ao quais o supervisor vai visualizando e selecionando). Tal dificuldade exige clareza no contrato de trabalho entre o supervisor e o supervisionando: como esta ocorrerá? A supervisão é voltada para todo o processo ou limitada à revisão de aplicação e levantamento de algum(ns) instrumento(s)? Caso seja voltada para todo o processo de avaliação, o supervisionando deverá enviar todos os protocolos aplicados. Isso será virtualmente ou fisicamente? Tais decisões irão influenciar na duração e consequentemente no valor da(s) supervisão(ões).

Alguns outros aspectos precisam ser levados em conta no estabelecimento da supervisão a distância, como a familiaridade dos usuários com o ambiente virtual, a necessidade de uma boa conexão para uso de videoconferência, e o uso de aplicativos ou plataformas que garantam segurança no compartilhamento de imagens de protocolos, documentos, vídeos e áudios de pacientes. É importante lembrar que esse cuidado não deveria ser restrito aos casos de supervisão online, mas também a casos de revisão de documentos feitos por supervisores que atendem seus supervisionandos presencialmente e que habitualmente os enviam por e-mail.

É útil ressaltar que supervisões esporádicas a distância entre supervisor e supervisionando que já se conhecem parecem ocorrer na prática, no entanto, ainda é incomum o estabelecimento de relacionamentos de supervisão exclusivamente remota. A teleconferência e a videoconferência em um ambiente de supervisão podem representar soluções para a necessidade de conveniência, disponibilidade e distância física. Os usuários devem ser instruídos a verificar as informações e credenciais dos supervisores antes de contratarem os serviços 
(Deane et al., 2015), assim como os supervisores precisam garantir as credenciais dos supervisionandos (serem psicólogos ou estudantes de psicologia).

Atualmente, ainda existem poucos estudos voltados à eficácia da supervisão em AP presencial (Vannucci et al., 2017), sendo mais comuns estudos e diretrizes sobre supervisão e treinamento em psicoterapia ou atendimento psicológico em saúde (APA, 2014). A isso se soma a necessidade de verificação das práticas de supervisão de AP online, visando compreender quais estratégias são mais efetivas na transmissão de conhecimento prático aos alunos, que possivelmente não se dão da mesma forma nos contextos presenciais e online.

\section{Considerações Finais}

A regulamentação de atividades psicológicas online contribuiu para ampliar as possibilidades de atuação do psicólogo brasileiro e desempenhou um papel central na adaptação ao novo cenário de trabalho imposto pelas restrições da pandemia da COVID-19, indicando que esse formato tende a se expandir e consolidar. No entanto, a complexidade do ambiente virtual suscita reflexões para a prática e o ensino de maneira ética e segura, em especial em uma área delicada como a AP.

Considerando os recursos disponíveis, é possível que a AP online seja administrável, neste momento, de maneira limitada e em situações pontuais. Reforça-se a necessidade de pesquisas que demonstrem evidências de validade e confiabilidade dos escores de uma maior variedade de testes para aplicação online ou remota, assim como o desenvolvimento de outras tecnologias que favoreçam e tornem segura a condução da AP online. Ainda, pondera-se que o psicólogo, antes de assumir a realização de um processo de AP online, considere sua capacitação teórica e técnica para a atividade, o que envolve também a competência para o uso de TIC.

No contexto do ensino de AP online, estratégias de segurança devem focar em reduzir a chance de disseminação de conteúdos relacionados a testes psicológicos e em aumentar a consciência ética entre estudantes e profissionais da Psicologia. Além do cuidado com o não compartilhamento de dados, o professor de AP deve prezar pela atualidade das informações oferecidas nos cursos de plataformas online.

Situações como a pandemia da COVID-19 demonstram como a supervisão a distância pode vir a ser útil para os profissionais da área da AP. Recomenda-se na supervisão as mesmas precauções com sigilo e restrições ao compartilhamento de dados da AP online. Novos estudos são necessários para a análise da eficácia da supervisão em AP tanto presencial como online.

Aproximar-se da tecnologia e da inovação, sem perder de vista os referenciais da ciência psicológica, é uma medida que tende a contribuir com a expansão e a especialização da área. É relevante ressaltar o papel dos professores e dos supervisores como guardiões da qualidade e da ética na área da AP online.

\section{Contribuição}

Todos os autores contribuíram integralmente na concepção, desenho, análise, interpretação dos dados e discussão dos resultados, bem como na revisão e aprovação da versão final do artigo.

\section{Referências}

American Psychological Association. (2013). Guidelines for the Practice of Telepsychology. American Psychologist, 68(9), 791-800. http://dx.doi.org/10.1037/a0035001 
American Psychological Association. (2014). Guidelines for Clinical Supervision in Health Service Psychology. American Psychologist, 70(1), 33-46. Retrieved from https://www.apa.org/about/policy/guidelines-supervision.pdf

American Psychological Association. (2020, May 1). How to do psychological testing via telehealth. American Psychological Association. Retrieved from https://uww.apaservices.org/practice/reimbursementhealth-codes/testing/psychological-telehealth

American Educational Research Association, American Psychological Association, \& National Council on Measurement in Education. (2014). Standards for educational and psychological testing. Washington: American Educational Research Association

Andrade, J. M., \& Valentini, F. (2018). Diretrizes para a construção de testes psicológicos: a Resolução CFP n 009/2018 em destaque. Psicologia: Ciência e Profissão, 38, 28-39. http://dx.doi.org/10.1590/1982-3703000208890

Bueno, J. M. H., \& Peixoto, E. M. (2018). Avaliação psicológica no Brasil e no mundo. Psicologia: Ciência e Profissão, 38, 108-121. http://dx.doi.org/10.1590/1982-3703000208878

Conselho Federal de Psicologia. (2005). Código de Ética Profissional do Psicólogo. Brasília: Autor. Recuperado de https:// site.cfp.org.br/wp-content/uploads/2012/07/codigo-de-etica-psicologia.pdf

Conselho Federal de Psicologia. (2018a). Resolução n 11, de 11 de maio de 2018. Regulamenta a prestação de serviços psicológicos realizados por meios de tecnologias da informação e da comunicação e revoga a Resolução CFP $n^{\circ}$ 11/2012. Brasília: Autor. Recuperado de https://site.cfp.org.br/wp-content/uploads/2018/05/resolu\%c3\%87\%c3\%83on\%c2\%ba-11-de-11-de-maio-de-2018.pdf

Conselho Federal de Psicologia. (2018b). Resolução n09, de 25 de abril de 2018. Estabelece diretrizes para a realização de Avaliação Psicológica no exercício profissional da psicóloga e do psicólogo, regulamenta o Sistema de Avaliação de Testes Psicológicos SATEPSI e revoga as Resoluções $n^{\circ}$ 002/2003, $n^{\circ}$ 006/2004 e $n^{\circ}$ 005/2012 e Notas Técnicas $n^{\circ}$ 01/2017 e 02/2017. Brasilia: Autor. Recuperado de http://satepsi.cfp.org.br/docs/resolu\%c3\%a7\%c3\%a3o-cfp-n\%c2\%ba-09-2018-com-anexo.pdf

Conselho Federal de Psicologia. (2019a). Nota Técnica nº 07/2019. Orienta psicólogas(os) sobre a utilização de testes psicológicos em serviços realizados por meio de tecnologias de informação e da comunicação. Brasília: Autor. Recuperado de https://site.cfp.org.br/wp-content/uploads/2019/10/nota-técnica-cfp-07.2019.pdf

Conselho Federal de Psicologia. (2019b). Resolução $n^{\circ}$ 06, de 29 de março de 2019. Institui regras para a elaboração de documentos escritos produzidos pela(o) psicóloga(o) no exercício profissional e revoga a Resolução CFP $n^{\circ}$ 15/1996, a Resolução CFP $n^{\circ}$ $07 / 2003$ e a Resolução CFP no 04/2019. Recuperado de http://www.in.gov.br/materia/-/asset_publisher/ kujrw0tzc2mb/content/id/69440957/do1-2019-04-01-resolucao-n-6-de-29-de-marco-de-2019-69440920

Conselho Federal de Psicologia. (2020a). Resolução nº 04, de 26 de março de 2020. Dispõe sobre regulamentação de serviços psicológicos prestados por meio de Tecnologia da Informação e da Comunicação durante a pandemia do COVID-19. Brasília: Autor. Recuperado de http://www.in.gov.br/en/web/dou/-/resolucao-n-4-de-26-de-marcode-2020-250189333

Conselho Federal de Psicologia. (2020b). Ofício-Circular n 63/2020/GTec/CG-CFP. Recomendações do CFP sobre a elaboração de documentos psicológicos para o Poder Judiciário no contexto da pandemia do novo coronavírus. Brasília: Autor. Recuperado de https://site.cfp.org.br/wp-content/uploads/2020/05/sei_cfp-0221879-of\%c3\%adcio-circular.pdf

Deane, F. P., Gonsalvez, C., Blackman, R., Saffioti, D., \& Andresen, R. (2015). Issues in the development of e-supervision in professional psychology: a review. Australian Psychologist, 50(3), 241-247. http://dx.doi.org/10.1111/ap.12107

Desmond, D. W., Tatemichi, T. K., \& Hanzawa, L. (1994). The Telephone Interview for Cognitive Status (TICS): reliability and validity in a stroke sample. International Journal of Geriatric Psychiatry, 9(10), 803-807. http://dx.doi.org/10.1002/ gps.930091006

Eckford, R. D., \& Barnett, D. L. (2016). Comparing paper-and-pencil and Internet survey methods conducted in a combatdeployed environment. Military Psychology, 28(4), 209-225. https://dx. doi.org/10.1037/mil0000118

Feijó, L. P., Silva, N. B., \& Benetti, S. P. C. (2018). Experiência e formação profissional de psicoterapeutas psicanalíticos na utilização das tecnologias de informação e comunicação. Psicologia: Ciência e Profissão, 38(2), 249-261. http:// dx.doi.org/10.1590/1982-3703003032017

Gouveia, V. (2018). Formação em avaliação psicológica: situação, desafios e diretrizes. Psicologia: Ciência e Profissão, 38, 74-86. http://dx.doi.org/10.1590/1982-3703000208641

Hallberg, S. C. M., \& Lisboa, C. S. M. (2016). Percepção e uso de tecnologias da informação e comunicação por psicoterapeutas. Temas em Psicologia, 24(4), 1297-1309. https://dx.doi.org/10.9788/TP2016.4-06

Hagstrom, S. L., \& Maranzan, K. A. (2019). Bridging the gap between technological advance and professional psychology training: a way forward. Canadian Psychology, 60(4), 281-289. http://dx.doi.org/10.1037/cap0000186

International Test Commission. (2005). International guidelines on computer-based and internet delivered testing. Lincoln: Author. Retrieved from https://www.intestcom.org/files/guideline_computer_based_testing.pdf 
International Test Commission. (2017). ITC guidelines for translating and adapting tests (2nd ed.). Lincoln: Author. Retrieved from https://www.intestcom.org/files/guideline_test_adaptation_2ed.pdf

Lustgarten, S. D., \& Elhai, J. D. (2018). Technology use in mental health practice and research: legal and ethical risks. Clinical Psychology: Science and Practice, 25(2), 1-10. http://dx.doi.org/10.1111/cpsp.12234

Luxton, D. D., Pruitt, L. D., \& Osenbach, J. E. (2014). Best practices for remote psychological assessment via telehealth technologies. Professional Psychology: Research and Practice, 45(1), 27-35. http://dx.doi.org/10.1037/a0034547

Marasca, A. R., Yates, D. B., Schneider, A. M. A., Feijó, L. P., \& Bandeira, D. R. (2020). Avaliação psicológica online: considerações a partir da pandemia do novo coronavírus (COVID-19) para a prática e o ensino no contexto à distância. Scielo Preprints. Versão 1. https://preprints.scielo.org/index.php/scielo/preprint/view/492/623

Martin, J. N., Millán, F., \& Campbell, L. F. (2020). Telepsychology practice: primer and first steps [Ahead of Print]. Practice Innovations. http://dx.doi.org/10.1037/pri0000111

McCord, C., Bernhard, P., Walsh, M., Rosner, C., \& Console, K. (2020). A consolidated model for telepsychology practice [Ahead of Print] Journal of Clinical Psychology. https://dx.doi.org/10.1002/jclp.22954

Ministério da Educação. (2019). Parecer CNE/CES no 1071/2019, aprovado em 4 de dezembro de 2019. Revisão das Diretrizes Curriculares Nacionais (DCNs) dos Cursos de Graduação em Psicologia e estabelecimento de normas para o Projeto Pedagógico Complementar (PPC) para formação de professores de psicologia. Brasília: Autor. Retirado de http://portal.mec.gov.br/index.php?option=com_docman\&view=download\&alias=139201-pces1071-19\&category slug=dezembro-2019-pdf\&ltemid=30192

Muniz, M. (2018). Ética na avaliação psicológica: velhas questões, novas reflexões. Psicologia: Ciência e Profissão, 38, 133-146. https://dx.doi.org/10.1590/1982-3703000209682

Oliveira, C. M., Bandeira, C. M., \& Giacomoni, C. H. (2019). Informatização e validação do instrumento tarefas preditoras de otimismo em crianças - TAPOC. Avaliação Psicológica, 18(2), 183-191. http://dx.doi.org/10.15689/ ap.2019.1802.14242.09

Poletti, B., Carelli, L., Solca, F., Lafronza, A., Pedroli, E., Faini, A.,... Silani, V. (2017). An eye-tracking controlled neuropsychological battery for cognitive assessment in neurological diseases. Neurological Sciences, 38, 595-603. http://dx.doi.org/10.1007/s10072-016-2807-3

Pritchard, A. E., Stephan, C. M., Zabel, T. A., \& Jacobson, L. A. (2017). Is this the wave of the future? Examining the psychometric properties of child behavior ratings administered online. Computers in Human Behavior, 70(4), 518-522. http://dx.doi.org/10.1016/j.chb.2017.01.030

Reips, U., Buchanan, T., Krantz, J., \& McGraw, K. (2016). Methodological challenges in the use of the internet for scientific research: ten solutions and recommendations. Studia Psychologica, 15(2), 139-148. http://dx.doi.org/10.21697/ sp.2015.14.2.09

Schmand, B. (2019). Why are neuropsychologists so reluctant to embrace modern assessment techniques? The Clinical Neuropsychologist, 33(2), 209-219. http://dx.doi.org/10.1080/13854046.2018.1523468

Schneider, A. M. A., Marasca, A. R., Dobrovolski, T. A. T., Muller, C. M., \& Bandeira, D. R. (2020). Planejamento da avaliação psicológica: implicações para a prática e para a formação. Scielo Preprints. Versão 1. https://doi.org/10.1590/ SciELOPreprints.521

Twist, M. L. C., Hertlein, K. M., \& Haider, A. (2016) Electronic communication in supervisory relationships: a mixed data survey. Contemporary Family Therapy, 38, 424-433. http://dx.doi.org/10.1007/s10591-016-9391-9

Vannucci, M. J., Whiteside, D. M., Saigal, S., Nichols, L., \& Hileman, S. (2017). Predicting supervision outcomes: what is different about psychological assessment supervision? Australian Psychologist, 52, 114-120. http://dx.doi.org/10.1111/ ap.12258

Wright, A. J., Brabender, V. M., \& Pade, H. (2019). The use of guidelines in assessment supervision. In A. J. Wright (Ed.), Essentials of psychological assessment (pp.19-48). New Jersey: John Wiley \& Sons.

Yates, D. B. (2016). Técnicas e modalidades de supervisão em psicodiagnóstico. In C. S Hutz, D. R. Bandeira, C. M. Trentini, \& J. F. Krug (Orgs.), Psicodiagnóstico (pp.194-203). Porto Alegre: Artmed.

Zaia, P., Oliveira, K. S., \& Nakano, T. C. (2018). Análise dos processos éticos publicados no jornal do Conselho Federal de Psicologia. Psicologia: Ciência e Profissão, 38(1), 8-21. http://dx.doi.org/10.1590/1982-3703003532016

Recebido: abril 29, 2020

Versão final: maio 15, 2020

Aprovado: maio 20, 2020 